World Health Organization. Guidelines for surveillance of drug resistance in tuberculosis. 4th Edn. http://whqlibdoc. who.int/publications/2009/9789241598675_eng.pdf Date last accessed: August 6, 2013. Date last updated: 2009.

Mesfin YM, Hailemariam D, Biadglign S, et al. Association between HIV/AIDS and multi-drug resistance tuberculosis: a systematic review and meta-analysis. PLoS ONE 2014; 9: e82235.

8 Suchindran S, Brouwer ES, Van Rie A. Is HIV infection a risk factor for multi-drug resistant tuberculosis? A systematic review. PLoS ONE 2009; 4: e5561.

9 Wells CD, Cegielski JP, Nelson LJ, et al. HIV infection and multidrug-resistant tuberculosis: the perfect storm. J Infect Dis 2007; 196: Suppl. 1, S86-S107.

10 Jenkins HE, Plesca V, Ciobanu A, et al. Assessing spatial heterogeneity of multidrug-resistant tuberculosis in a highburden country. Eur Respir J 2012; 42: 1291-1301.

11 Moonan PK, Teeter LD, Salcedo K, et al. Transmission of multidrug-resistant tuberculosis in the USA: a crosssectional study. Lancet Infect Dis 2013; 13: 777-784.

12 Porco TC, Oh P, Flood JM. Antituberculosis drug resistance acquired during treatment: an analysis of cases reported in California, 1994-2006. Clin Infect Dis 2013; 56: 761-769.

13 Falzon D, Jaramillo E, Wares F, et al. Universal access to care for multidrug-resistant tuberculosis: an analysis of surveillance data. Lancet Infect Dis 2013; 13: 690-697.

14 Nardell E, Dharmadhikari A. Turning off the spigot: reducing drug-resistant tuberculosis transmission in resourcelimited settings. Int J Tuberc Lung Dis 2010; 14: 1233-1243.

15 Getahun H, Gunneberg C, Granich R, et al. HIV infection-associated tuberculosis: the epidemiology and the response. Clin Infect Dis 2010; 50: S201-S207.

\title{
Rapid diagnosis of tuberculosis using ex vivo host biomarkers in sputum
}

To the Editor:

Tuberculosis continues to be a major public health problem in developing countries [1]. One of the roadblocks in reducing tuberculosis transmission is the lack of accurate laboratory-free diagnostic tests for use at the point of care. If tuberculosis is to be eliminated, we need a robust, low-cost and safe point-of-care diagnostic test, which in turn requires identification of appropriate biomarkers [2]. Rapid tests based on microfluidics (lateral flow tests) hold great promise for tuberculosis diagnostics. They are easy to use, cheap, provide an answer within minutes, do not require specialised equipment and are stable at room temperature, making them ideal for use in high tuberculosis burden, resource-poor settings. To date, however, no such test has been developed for tuberculosis due to lack of sensitivity related to the markers and/or sample type. Development of tests based on host biomarkers requires evaluation of different sample types [3-5] and markers other than interferon (IFN)- $\gamma$ [5] to provide differential diagnosis of active tuberculosis, latent infection and other respiratory disorders. We have previously shown that a combination of three host factors in pleural fluid resulted in $96 \%$ correct classification of tuberculosis among other respiratory diseases (ORD) (including bacterial pneumonia) regardless of HIV status [6]. However, this sample type is not easy to obtain and we therefore wanted to determine if we could use ex vivo sputum, which is noninvasive and easy to obtain in adult pulmonary tuberculosis patients.

Subjects were consecutively recruited from the outpatient clinic and ward at the Medical Research Council Unit, Fajara, the Gambia. All subjects were adults ( $\geqslant 18$ years of age) with symptoms suggestive of tuberculosis. Subjects were subsequently classified into two groups: those with culture-confirmed tuberculosis and those with ORD. 75\% of the tuberculosis and 50\% of the ORD group were positive by the IFN- $\gamma$ QuantiFERON test (Qiagen, Hilden, Germany). Samples were collected concomitantly from the same patient. Serum was collected using serum Vacutainers (BD, Franklin Lakes, NJ, USA) following centrifugation and saliva was collected using a passive drool technique. $1 \mathrm{~mL}$ of fresh sputum was digested for $15 \mathrm{~min}$ at room temperature with $0.1 \%$ dithiothreitol. An equal volume of PBS was added, the samples were centrifuged $(600 \times g$ for $5 \mathrm{~min})$, and the supernatants were collected and stored at $-20^{\circ} \mathrm{C}$. Undiluted heparinised blood $(450 \mu \mathrm{L}$ per well) was stimulated with purified protein derivative (PPD) (Statens Serum Institut, Copenhagen, Denmark) or ESAT-6 (6-kDa early secreted antigen)/CFP-10 (10-kDa culture filtrate protein) at a final concentration of $10 \mu \mathrm{g} \cdot \mathrm{mL}^{-1}$. After $24 \mathrm{~h}$ incubation (at $37^{\circ} \mathrm{C}$ and $5 \%$ carbon dioxide), supernatants were harvested and analysed by multiplex cytokine array. Samples were analysed using either a custom 13-plex (stimulated blood) or 27-plex Bio-Plex (serum, saliva and sputum) pre-mixed cytokine/ chemokine kits according to the manufacturer's instructions (Bio-Rad, Nazareth-Eke, Belgium). Levels of cytokines in tuberculosis and non-tuberculosis subjects were analysed using the Mann-Whitney U-test. 
Logistic regression and receiver operating curve analyses were performed, and adjusted for age and sex. Graphs were generated using GraphPad Prism version 6.0 (Software MacKiev, Boston, MA, USA) and statistical analysis with SPSS version 20 (IBM, Armonk, NY, USA). p-values $\leqslant 0.035$ were considered significant to account for false-discovery rates.

Following overnight whole-blood stimulation, $10-\mathrm{kDa}$ IFN- $\gamma$-inducible protein (IP10) and monocyte chemoattractant protein (MCP)-1 levels were high in both groups following all stimulations, while transforming growth factor (TGF)- $\alpha$, epidermal growth factor and vascular endothelial growth factor (VEGF) levels were low (fig. 1a). IP10, CD40 ligand (CD40L), TGF- $\alpha$, tumour necrosis factor (TNF)- $\alpha$ and IFN- $\gamma$ were all significantly higher in subjects with confirmed tuberculosis compared with ORD in unstimulated samples $(\mathrm{p}=0.0005, \mathrm{p}=0.0089, \mathrm{p}=0.0020, \mathrm{p}=0.0016$ and $\mathrm{p}=0.0313$, respectively). Following background subtraction, the main differences were observed in the PPD-stimulated samples, with higher levels of CD40L, IL-10 and TGF- $\alpha$ in tuberculosis compared with non-tuberculosis subjects $(\mathrm{p}=0.0089$, $p=0.0034$ and $p<0.0001$, respectively) but lower levels of IFN- $\gamma$, interleukin IL) -2 and macrophage inflammatory protein (MIP) $-1 \beta(\mathrm{p}=0.0313, \mathrm{p}=0.0040$ and $\mathrm{p}=0.035$, respectively). Using logistic regression analysis, the best classification was achieved following PPD stimulation with a combination of CD40L, TGF- $\alpha$ and IL-10, giving $89 \%$ correct classification of tuberculosis or ORD. Digested sputum showed surprisingly high levels of cytokines ex vivo compared with both saliva and serum (fig. 1b). Levels of IL-4, IL-5, IL-10, IL-13, IL-7, IL-8, IL-12 ${ }^{\mathrm{p} 70}$ and MIP-1 $\beta$ were all significantly higher in sputum compared with both saliva and serum (illustrated in fig. $1 \mathrm{~b}$ by IL-7 and IL-8), while IL-1 $\beta$, IL-17, granulocyte colony-stimulating factor (G-CSF), granulocyte-macrophage colony-stimulating factor, MCP-1 and TNF- $\alpha$ were significantly higher in both saliva and sputum compared with serum (illustrated in fig. 1b by G-CSF and MCP-1). IL- 6 was the only cytokine that was lower in saliva compared with both serum $(\mathrm{p}<0.01)$ and sputum $(\mathrm{p}<0.0001)$, with no difference between serum and sputum (data not shown), and no difference in IFN- $\gamma$ levels seen between the three sample types (fig. 1b).

We next compared cytokine levels in ex vivo sputum from tuberculosis and non-tuberculosis (ORD) subjects (fig. 1c). Interestingly, we found no difference in pro-inflammatory cytokines (i.e. TNF- $\alpha$, IFN- $\gamma$ and IP10) but significantly lower levels of IL-10 ( $\mathrm{p}=0.004)$, IL-13 $(\mathrm{p}=0.003)$ and IL-15 $(\mathrm{p}=0.022)$ were found in sputum from tuberculosis compared with non-tuberculosis subjects (fig. 1c). Additionally, the innate cytokines IL-1 receptor antagonist, G-CSF and VEGF were all significantly lower $(p=0.005, p=0.004$ and $\mathrm{p}=0.030$, respectively), while fibroblast growth factor (FGF) was significantly higher in tuberculosis compared with non-tuberculosis subjects (median (interquartile range) $287(40-764) \mathrm{pg} \cdot \mathrm{mL}^{-1}$ and 2.2 $(0-325) \mathrm{pg} \cdot \mathrm{mL}^{-1}$, respectively; $\mathrm{p}=0.007$ ) (fig. 1c). Levels of FGF alone gave $74 \%$ correct classification of tuberculosis (sensitivity (95\% CI) 78\% (56-93\%) and specificity 67\% (47-83\%)). Logistic regression (with age and sex adjustment) revealed a combination of IL-13, FGF and IFN- $\gamma$ resulted in $96 \%$ correct classification of tuberculosis (sensitivity $85 \%$ and specificity $96 \%$ ).

Alongside high sensitivity and specificity, one of the main criteria for development of a lateral-flow based point-of-care test for tuberculosis is time to results [7]. We found levels of host biomarkers in ex vivo sputum were significantly higher than levels measured following in vitro antigen-stimulated blood cultures, thus reducing time to diagnosis. Sputum is routinely used for microbiological detection of Mycobacteriun tuberculosis and is easily obtainable, making it an ideal sample type for development of a lateral flow test for tuberculosis. Interestingly, no difference in Th1 cytokine levels were observed between tuberculosis and non-tuberculosis subjects using ex vivo sample types. This may be due to the level of latent $M$. tuberculosis infection in the non-tuberculosis group (50\% were positive by QuantiFERON test). Conversely, the Th2 cytokines IL-10 and IL-13 were both significantly lower in tuberculosis compared with non-tuberculosis sputa, indicating a bias towards Th1 responses in subjects with tuberculosis. In a previous study in Brazil, levels of IFN- $\gamma$ in sputum were shown to equate with treatment response [8] but we did not assess that in the present study. G-CSF is required for neutrophil recruitment and was found to be significantly lower in sputum from tuberculosis compared with non-tuberculosis subjects in our study. This is interesting, as neutrophils are a major component of the protective immune response to tuberculosis [9] and G-CSF administration has been shown to increase response to tuberculosis therapy [10]. While most factors were lower in tuberculosis compared with non-tuberculosis samples, FGF was significantly higher. Interestingly, M. tuberculosis-infected fibroblasts lose their capacity for antigen presentation, suggesting that M. tuberculosis may evade T-helper immune surveillance by infecting fibroblasts, thereby resulting in bacterial persistence [11].

We only analysed subjects with culture-confirmed tuberculosis and, of these, only three were smear negative (14\%), so it is difficult at this stage to determine sensitivity in smear-negative subjects. However, $96 \%$ correct classification of tuberculosis using a combination of FGF, IL-13 and IFN- $\gamma$ from sputum is significantly higher than results reported from current blood-, breath- or urine-based tests $[4,7]$. Thus, our 


\begin{tabular}{|c|c|c|c|c|c|c|c|c|c|}
\hline \multirow[t]{2}{*}{ a) } & \multicolumn{3}{|c|}{ Control } & \multicolumn{3}{|c|}{ ESAT-6/CFP-10 } & \multicolumn{3}{|c|}{ PPD } \\
\hline & TB & ORD & $p$-value & TB & ORD & p-value & TB & ORD & $p$-value \\
\hline CD40L & 2949 & 1728 & 0.089 & 3171 & 1869 & 0.0063 & 3647 & 1946 & 0.0089 \\
\hline EGF & 50 & 46 & NS & 57 & 39 & NS & 54 & 34 & NS \\
\hline MIP-1 $\beta$ & 136 & 95 & NS & 3463 & 4740 & NS & 2566 & 4251 & 0.0351 \\
\hline TGF- $\alpha$ & 6 & 3 & 0.0020 & 23 & 4 & $<0.0001$ & 30 & 10 & $<0.0001$ \\
\hline VEGF & 81 & 3 & NS & 34 & 13 & NS & 76 & 3 & NS \\
\hline IFN- $\gamma$ & 6 & 3 & 0.0313 & 204 & 120 & NS & 443 & 600 & 0.0313 \\
\hline IL-10 & 3 & 3 & NS & 34 & 59 & NS & 314 & 90 & 0.0034 \\
\hline IL-2 & 3 & 3 & NS & 21 & 25 & NS & 205 & 514 & 0.0040 \\
\hline IP10 & 4353 & 1127 & 0.0005 & 10000 & 10000 & NS & 10000 & 10000 & NS \\
\hline MCP-1 & 1065 & 1221 & NS & 7838 & 9908 & NS & 9378 & 10000 & NS \\
\hline TNF- $\alpha$ & 6 & 3 & 0.016 & 163 & 133 & NS & 103 & 116 & NS \\
\hline
\end{tabular}

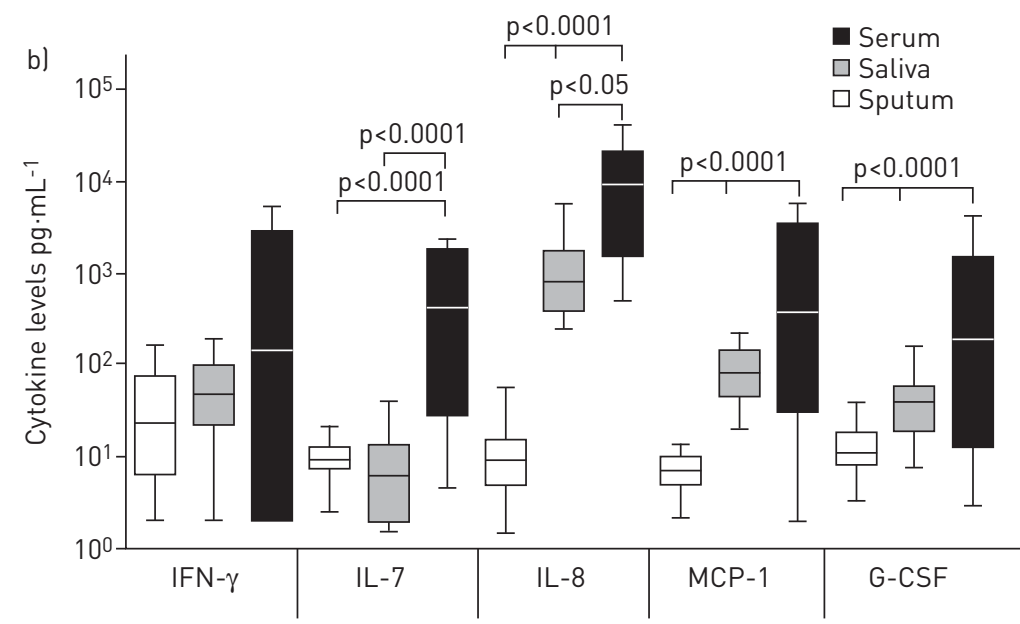

c)

\begin{tabular}{|c|c|c|c|c|c|c|c|c|c|c|c|c|c|c|c|c|c|c|}
\hline & $\mid \mathrm{IL-1} \beta$ & IL-1ra & IL-4 & IL-6 & IL-7 & IL-8 & IL-10 || & $\mid L-12^{p^{7}}$ & 70 IL-13 & FGF & G-CSF & $\mathrm{IFN}-\gamma$ & IP10 & MCP-1I & MIP-1 $1 \beta$ & PDGF & TNF-c & VEGF \\
\hline TB & 2499 & 3458 & 39 & 41 & 423 & 9288 & 7 & 268 & 25 & 287 & 189 & 144 & 5064 & 373 & 702 & 44 & 11 & 11 \\
\hline ORD & 1577 & 1092 & 7 & 98 & 357 & 14629 & 210 & 802 & 155 & 2 & 1805 & 659 & 9164 & 1186 & 473 & 150 & 64 & 64 \\
\hline$p$-value & NS & 0.0005 & NS & NS & NS & NS & 0.0004 & NS & 0.0003 & 0.0071 & 0.0041 & NS & NS & NS & NS & NS & NS & NS \\
\hline
\end{tabular}

Low $\longrightarrow$ High

Analyte levels $\mathrm{pg} \cdot \mathrm{mL}^{-1}$

FIGURE 1 a) Heat map of cytokine profiles following overnight stimulation of whole blood with tuberculosis antigens. Median values are presented. b) Comparison of cytokine levels in serum, saliva and sputum from tuberculosis patients $(n=25, n=20$ and $n=23$, respectively). Note that values shown are not adjusted for dilution of the sputum cytokines during digestion (four-fold). Boxes indicate interquartile ranges, horizontal lines indicate medians and whiskers indicate 5-95\% range. Data were anlysed using the Kruskal-Wallis test followed by Dunn's multiple comparison. p-values $\leqslant 0.035$ were considered significant and are indicated. c) Heat map of cytokine levels in ex vivo sputum. Median values are presented for subjects with tuberculosis $(n=23)$ and those with other respiratory disorders (ORD) $(n=29)$. Data were anlysed using a Mann-Whitney U-test. p-values $\leqslant 0.035$ were considered significant and are indicated. Control: no antigen; ESAT-6: 6-kDa early secreted antigen; CFP-10: 10-kDa culture filtrate protein; PPD: purified protein derivative; TB: culture-confirmed tuberculosis; CD40L: CD40 ligand; EGF: epidermal growth factor; MIP: macrophage inflammatory protein; TGF: transforming growth factor; VEGF: vascular endothelial growth factor; IFN: interferon; IL: interleukin; IP10: $10-\mathrm{kDa}$ IFN- $\gamma$-inducible protein; MCP: monocyte chemoattractant protein; TNF: tumour necrosis factor; NS: nonsignificant; G-CSF: granulocyte colony-stimulating factor; IL-1Ra: IL-1 receptor antagonist; FGF: fibroblast growth factor; PDGF: platelet-derived growth factor.

findings hold promise for future development of a rapid lateral flow-based diagnostic test for tuberculosis that is applicable for use in resource-limited settings. Tuberculosis elimination will never be reached in developing countries without a synergistic approach including development of better diagnostics that are fast and affordable $[2,7,12]$. 

point-of-care test http://ow.ly/tsx2S

Martin O.C. Ota ${ }^{1}$, Joseph F. Mendy ${ }^{1}$, Simon Donkor ${ }^{1}$, Toyin Togun ${ }^{1}$, Mohammed Daramy ${ }^{1}$, Marie P. Gomez ${ }^{1}$, Novel N. Chegou ${ }^{2}$, Abdou K. Sillah ${ }^{1}$, Olumuyiwa Owolabi ${ }^{1}$, Beate Kampmann ${ }^{1}$, Gerhard Walzl ${ }^{2}$ and Jayne S. Sutherland ${ }^{1}$

${ }^{1}$ Vaccinology Theme, Medical Research Council Unit, Fajara, the Gambia. ${ }^{2}$ DST/NRF Centre of Excellence for Biomedical Tuberculosis Research and MRC Centre for Molecular and Cellular Biology, Division of Molecular Biology and Human Genetics, Dept of Biomedical Sciences, Faculty of Medicine and Health Sciences, Stellenbosch University, Tygerberg, South Africa.

Correspondence: Jayne S. Sutherland, MRC Unit, Atlantic Boulevard, Banjul, the Gambia. E-mail: jsutherland@mrc.gm

Received: Nov 072013 | Accepted after revision: Jan 282014 | First published online: March 132014

Support statement: This research was funded by European and Developing Countries Clinical Trials Partnership (grant number 09.32040.011) and the UK Medical Research Council. The funders had no role in study design, data collection and analysis, decision to publish, or preparation of the manuscript.

Conflict of interest: Disclosures can be found alongside the online version of this article at www.erj.ersjournals.com

\title{
References
}

World Health Organization. WHO Global Tuberculosis Report 2013. www.who.int/tb/publications/factsheet_ global.pdf Date last updated: 2013. Date last accessed: February 28, 2014.

2 Weyer K, Mirzayev F, Migliori GB, et al. Rapid molecular TB diagnosis: evidence, policy making and global implementation of Xpert MTB/RIF. Eur Respir J 2013; 42: 252-271.

3 Albanna AS, Bachmann K, White D, et al. Serum lipids biomarkers for therapeutic monitoring of latent tuberculosis infection. Eur Respir J 2013; 42: 547-550.

4 Minion J, Leung E, Talbot E, et al. Diagnosing tuberculosis with urine lipoarabinomannan: systematic review and meta-analysis. Eur Respir J 2011; 38: 1398-1405.

5 Chegou NN, Heyckendorf J, Walzl G, et al. Beyond the IFN- $\gamma$ horizon: biomarkers for immunodiagnosis of infection with M. tuberculosis. Eur Respir J 2014; 43: 1472-1486.

6 Sutherland JS, Garba D, Fombah AE, et al. Highly accurate diagnosis of pleural tuberculosis by immunological analysis of the pleural effusion. PLoS One 2012; 7: e30324.

7 Batz HG, Cook GS, Reid SD. Towards lab-free tuberculosis diagnostics. www.msfaccess.org/sites/default/files/ MSF_assets/TB/Docs/TB_Report_TowardsLabFreeTBDX_2011_ENG.pdf

8 Ribeiro-Rodrigues R, Resende Co T, Johnson JL, et al. Sputum cytokine levels in patients with pulmonary tuberculosis as early markers of mycobacterial clearance. Clin Diagn Lab Immunol 2002; 9: 818-823.

9 Yang CT, Cambier CJ, Davis JM, et al. Neutrophils exert protection in the early tuberculosis granuloma by oxidative killing of mycobacteria phagocytosed from infected macrophages. Cell Host Microbe 2012; 12: 301-312.

10 Cormican LJ, Schey S, Milburn HJ. G-CSF enables completion of tuberculosis therapy associated with iatrogenic neutropenia. Eur Respir J 2004; 23: 649-650.

11 M.ariotti S, Sargentini V, Pardini M, et al. Mycobacterium tuberculosis may escape helper T cell recognition by infecting human fibroblasts. Hum Immunol 2013; 74: 722-729.

12 Diel R, Loddenkemper R, Zellweger JP, et al. Old ideas to innovate tuberculosis control: preventive treatment to achieve elimination. Eur Respir J 2013; 42: 785-801.

\section{Serial testing using interferon- $\gamma$ release assays in nursing students in India}

\author{
To the Editor:
}

We have previously shown that Indian healthcare workers have higher prevalence of latent tuberculosis infection (LTBI) and are at increased risk for new infection [1-4]. Interferon- $\gamma$ release assays (IGRAs) have been introduced as an alternative to the tuberculin skin test (TST) for diagnosing LTBI in healthcare workers and other high-risk groups. They have logistical advantages over the TST and will not cross-react with the bacille Calmette Guérin vaccine. IGRAs are now being widely used for screening healthcare workers [5], yet recent reports indicate that switching from TST to IGRAs for the serial testing of healthcare workers may result in increased rates of test conversions and reversions [3, 6-8]. Most of these studies are from low tuberculosis (TB) incidence settings, with limited opportunity for nosocomial TB exposure; as a result, the increased conversion rates are considered false-positive test conversions, making it difficult for clinicians to 